\title{
HOMOLOGY PROPERTIES OF ARBITRARY SUBSETS OF EUCLIDEAN SPACES
}

\author{
BY \\ SAMUEL KAPLAN
}

Introduction. The study of the homology properties of topological spaces by means of coverings, first clearly formulated by Cech [3](1), has been successfully applied to compact spaces by( $\left.{ }^{2}\right) \mathrm{him}$ and other topologists. The large body of theory which has grown up around this work, however, deals almost exclusively with finite coverings, and these alone are not a sufficient tool for non-compact spaces. This was graphically demonstrated by Dowker [4], who showed that under finite coverings the linear continuum has nonbounding one-dimensional Cech cycles. Essentially the trouble is that we cannot expect to make a thorough analysis of a topological space, as far as homology is concerned, without using a complete family of coverings; and for a non-compact space the finite coverings do not form a complete family.

Thus, if we are going to extend our studies to spaces other than the compact, a basic problem in the study of each type of space will be to find the simplest possible complete family of coverings for that type. In the present paper we take up the class of separable metric spaces. Since these include all the subsets of Euclidean spaces, they constitute a large and important collection. The finite coverings of course do not form a complete family for such spaces. Next to the finite, the simplest coverings are the countable star-finite, and we show in $\$ 1$ that these do form a complete family.

In $\$ 2$ we consider Cech cycles on compact subsets of a separable metric space, and compare the property of bounding on a compact subset with the (more general) property of bounding in the space as a whole. We obtain a topologically invariant type of bounding for Vietoris cycles which is equivalent to the latter. In $\$ 3$ we consider the question of obtaining the homology properties of an arbitrary subset $A$ of a separable metric space $R$ by means of open sets of $R$. As is well known, if $A$ is closed in $R$, we can obtain all the homology properties of $A$ by using coverings of $R$ and considering the parts of such coverings which meet $A$. If $A$ is not closed, this is no longer true. As is shown in $\S 3$, the general solution is to use not coverings of $R$ but coverings of all the neighborhoods of $A$ in $R$.

In $\$ 4$ we establish the isomorphism between the Cech homology groups

Presented to the Society, April 18, 1942, under the title Homologies in metric separable spaces; received by the editors August 30, 1946.

( $)$ Numbers in brackets refer to the references cited at the end of the paper.

( ${ }^{(2)}$ A space $R$ will be called compact if for every covering of $R$ by open sets, there is a finite number of the open sets which covers $R$. 
and the classical homology groups of an infinite complex. We then show that if $A$ is an arbitrary subset of a polyhedral complex, its homology properties can be studied by means of singular cycles in the neighborhoods of $A$. We also obtain the important property that a Cech cycle on a compact subset of $A$ bounds in $A$ if and only if it bounds on some compact subset in every neighborhood of $A$. In $\$ 5$ we study the duality properties of arbitrary subsets of the $n$-sphere. We obtain generalizations of the Alexander duality to such arbitrary subsets. Using these we also obtain some relations between the dimension of an arbitrary subset and the homology properties of its complement, including the accessibility of its points from the complement. In $\S 6$ we show that if $A$ is an arbitrary subset of the $n$-sphere, the $(n-1)$-dimensional homology properties of $A$ can be obtained by considering only finite coverings of $A$.

1. The covering theorem. By a covering of a topological space $R$, we shall mean a collection $\mathfrak{U}=\{U\}$, where the $U$ 's are open sets of $R$ and $U U=R$. The $U$ 's are called elements of $\mathfrak{u}$. Unless otherwise stated, there will be no restriction on the number of elements in a covering. Given the covering $\mathfrak{U}=\{U\}$, a covering $\mathfrak{B}=\{V\}$ is called a refinement of $\mathfrak{u}$-in symbols $\mathfrak{B}>\mathfrak{U}-$ if every $V$ is contained in some $U$. A collection of coverings $\left\{\mathfrak{u}^{\prime}\right\}$ is called a complete family of coverings for $R$ if for every covering $\mathfrak{u}$ there is a $\mathfrak{u}^{\prime}>\mathfrak{u}$.

A covering is called finite if it contains only a finite number of elements. It is called star-finite if each element intersects only a finite number of the elements. A finite covering is of course star-finite. Finally, a covering is called countable if it contains a countable number of elements $\left({ }^{8}\right)$.

ThEOREM 1. The countable star-finite coverings form a complete family for separable metric spaces.

Proof. By Urysohn's metrization theorem, a separable metric space is homeomorphic to a subset of the Hilbert cube, which is a compact metric space. It is therefore sufficient to prove the theorem for an arbitrary subset of a compact metric space. Let $R$ be a compact metric space, $A$ an arbitrary subset of $R$, and $\mathfrak{U}=\{U\}$ a covering of $A$ by open subsets of $A$. For each $U$, let $U^{\prime}$ be an open subset of $R$ such that $U^{\prime} \cap A=U$. (For example $U^{\prime}$ might be defined by $U^{\prime}=R-$ Closure $(A-U)$.) Then $G=U U^{\prime}$ is an open subset of $R$ containing $A$, and $\mathfrak{u}^{\prime}=\left\{U^{\prime}\right\}$ is a covering of $G$.

We shall first obtain a countable star-finite covering of $G$ which is a refinement of $\mathfrak{u}^{\prime}$. If $G$ is closed in $R$, we simply apply the Borel theorem. If not, it is the union of an expanding sequence of open sets, $G=\cup_{i=0}^{\infty} G_{i}$, such that $\bar{G}_{i} \subset G_{i+1}$ (we shall take $G_{0}=0$ ). For each $i>0, \bar{G}_{i}$ is compact and contained in $G$. Therefore it is covered by a finite number of the $U^{\prime}$; denote them by

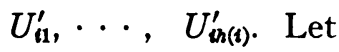

(8) By countable we shall mean finite or countably infinite. 


$$
V_{i j}^{\prime}=U_{i j}^{\prime} \cap\left(G_{i+1}-\bar{G}_{i-1}\right) \text {. }
$$

Then $\left\{V_{i j}^{\prime}\right\}(i=1,2, \cdots ; j=1,2, \cdots, h(i))$ is the required countable starfinite refinement of $\mathfrak{u}^{\prime}$. Let us denote it by $\mathfrak{B}^{\prime}=\left\{V_{m}^{\prime}\right\}$.

Now let $V_{m}=V_{m}^{\prime} \cap A$. Then $\mathfrak{B}=\left\{V_{m}\right\}$ is a countable star-finite covering of $A$ which is a refinement of $\mathfrak{u}$. This ccmpletes the proof.

Two coverings, $\mathfrak{U}=\{U\}$ and $\mathfrak{B}=\{V\}$, will be called isomorphic if there is a one-to-one correspondence between their elements such that if $U_{1}, \cdots, U_{h}$ are the images of $V_{1}, \cdots, V_{h}$ respectively, then $\bigcap_{i=1}^{h} U_{i} \neq 0$ implies $\bigcap_{i=1}^{h} V_{i} \neq 0$, and conversely. Given a covering $\mathfrak{U}=\{U\}$, by $\overline{\mathfrak{u}}$ we shall denote the closed covering $\{\bar{U}\}$. A covering $\mathfrak{u}$ such that $\mathfrak{u}$ is isomorphic to $\overline{\mathfrak{u}}$ will be called closure-isomorphic.

We now demonstrate a stronger form of the covering theorem.

(1.1) The closure-isomorphic, countable, star-finite coverings form a complete family for metric separable spaces.

This is a corollary of Theorem 1 and the following theorem.

(1.2) If a topological space $R$ is normal and $\mathfrak{U}$ is a countable star-finite covering of $R$, then there is a covering $\mathfrak{B}>\mathfrak{U}$ which is closure-isomorphic and isomorphic to $\mathfrak{U}$ (hence $\mathfrak{B}, \overline{\mathfrak{B}}$, and $\mathfrak{\mathfrak { U }}$ are all isomorphic).

Proof. Let $\mathfrak{U}=\left\{U_{i}\right\}$. We shall obtain a covering $\mathfrak{B}=\left\{V_{i}\right\}$ such that

(i) $\bar{V}_{i} \subset U_{i}$,

(ii) if $\bigcap_{j=1}^{h} U_{i(j)} \neq 0$, then $\bigcap_{j=1}^{h} V_{i(j)} \neq 0$.

Then $\mathfrak{B}$ will be the required refinement. We define the elements of $\mathfrak{B}$ inductively. $U_{1}$ intersects only a finite number of the rest of the $U_{i}$, therefore there is only a finite number of nonvacuous intersections of the form

$$
U_{1} \cap U_{i(1)} \cap \cdots \cap U_{i(h)} \quad(i(1), \cdots, i(h) \text { running through } 2,3, \cdots) .
$$

Denote these interscctions by $G_{j}(j=1, \cdots, t)$ and let $x_{j} \in G_{j}(j=1, \cdots, t)$. The set $\left(U_{1}-U_{i=1} U_{i}\right) \cup\left(U_{j=1}^{t} x_{j}\right)$ is closed and lies in $U_{1}$. Therefore, from the normality of $R$, it is contained in an open set $V_{1}$ such that $\bar{V}_{1} \subset U_{1}$. The covering $\left\{V_{1}, U_{2}, U_{3}, \cdots\right\}$ is obviously isomorphic to $\mathfrak{u}$.

Now assume we have defined $V_{1}, \cdots, V_{n-1}$ such that $\bar{V}_{i} \subset U_{i}(i=1, \cdots$, $n-1)$ and the covering

$$
\left\{V_{1}, \cdots, V_{n-1}, U_{n}, U_{n+1}, \cdots\right\}
$$

is isomorphic to $\mathfrak{u}$. We then define $V_{n}$ relative to $U_{n}$ and (iii) by the same method we used in defining $V_{1}$ relative to $U_{1}$ and $\mathfrak{u}$. We thus obtain a covering $\left\{V_{i}\right\}$ satisfying (i) and (ii), which proves the theorem.

2. General bounding and compact bounding. In the homology theory of this paper, all coefficients will be from some fixed field, and except when it becomes necessary, no mention is henceforth made of the field of coefficients. 
Let $R$ be a topological space and $\mathfrak{u}$ a covering of $R$. We shall denote the nerve $\left.{ }^{4}\right)$ of $\mathfrak{u}$ by $K(\mathfrak{U})$. We shall speak of chains of $K(\mathfrak{U})$ as chains of $\mathfrak{u}$, and of homologies in $K(\mathfrak{U})$ as homologies in $\mathfrak{u}$. We shall restrict ourselves entirely to finite chains and cycles. If $\mathfrak{B}>\mathfrak{U}$ and $\pi$ is a projection of $K(\mathfrak{B})$ into $K(\mathfrak{U})$, we shall say that $\pi$ is a projection from $\mathfrak{B}$ into $\mathfrak{U}$. If $Z^{k}(\mathfrak{B})$ is a cycle of $\mathfrak{B}$, we shall denote its projection in $\mathfrak{U}$ by $\pi Z^{k}(\mathfrak{B})$.

A $C$ - $k$-cycle, or $k$-dimensional Čech cycle, will be a collection $C^{k}=\left\{Z^{k}(\mathfrak{U})\right\}$, where $\mathfrak{U}$ runs through all the coverings of $R$ and for every $\mathfrak{B}>\mathfrak{U}$ and $\pi$ a projection from $\mathfrak{B}$ into $\mathfrak{U}, \pi Z^{k}(\mathfrak{B}) \sim Z^{k}(\mathfrak{U})$ in $\mathfrak{U}[3]$. $C^{k}$ will be said to bound, or be homologous to zero, in $R$-in symbols, $C^{k} \sim 0$ in $R$-if $Z^{k}(\mathfrak{U}) \sim 0$ in $\mathfrak{U}$ for all coverings $\mathfrak{u}$ of $R$. As is well known, we may restrict ourselves, in the above definitions, to members of a complete family of coverings.

Now let $A$ be a subset of $R$. If $\mathfrak{u}=\{U\}$ is a covering of $R$, the collection of those elements of $\mathfrak{U}$ which intersect $A$ will be called an external covering of $A$, with the understanding that $U_{0}, \cdots, U_{k}$ form a simplex in the nerve of this covering only if $\bigcap_{i=0}^{k} U_{i}$ intersects $A$. We shall speak of the chains in this external covering as being on $A$, and similarly for the homologies. The set of all external coverings of $A$ gives us Cech cycles, which we shall call Cech cycles on $A$. If a Cech cycle on $A$ bounds in all the external coverings of $A$, we shall say it bounds on $A$. It is easily shown( $\left.{ }^{5}\right)$ that if $A$ is closed in $R$, these Cech homology groups defined by the external coverings are isomorphic with the usual ones, that is, those obtained by coverings of $A$ made up of open subsets of $A$-the latter we shall call internal coverings.

In particular, if $R$ is metric and $A$ is a compact subset, the $k$-dimensional Cech homology group of $A$ obtained by external coverings, the one obtained by internal coverings, and the $k$-dimensional Vietoris homology group [ 1 , chap. IV, §5] of $A$ are all three isomorphic, for all $k$. We shall call a Čech cycle given for such a set $A$ by external coverings, a corresponding one (determined up to a homology) given by internal coverings, and a corresponding Vietoris cycle (also determined up to a homology) counterparts of each other. In general, we shall not distinguish between counterpart external and internal Cech cycles; that is, we shall merely write $C^{k}=\left\{Z^{k}(\mathfrak{u})\right\}$, and let $\mathfrak{u}$ run through both external and internal coverings. It should be noted that only Cech cycles on compact subsets of $R$ have Vietoris counterparts. We shall call a Cech cycle which is on a compact subset of a space a compact Cech cycle. (As opposed to this, if we are not concerned with whether a Cech cycle lies on a compact set or not, and wish to emphasize this, we shall refer to it as a general Cech cycle.)

$\left({ }^{4}\right)$ Given a covering of a space, the nerve of the covering is the abstract simplicial complex defined as follows: Every element of the covering is a vertex of the nerve and for each natural number $k$ every set of $k+1$ elements with a nonvacuous intersection forms a $k$-simplex of the nerve.

(5) Cf. [3, chap. 3]. Cech's argument holds for arbitrary coverings. 
Between a compact Cech cycle and any of its Vietoris counterparts there exists the following easily proved relation.

(2.1) Let $A$ be a compact subset of a metric space $R, C^{k}=\left\{Z^{k}(\mathfrak{U})\right\}$ and ( $\left.{ }^{\circ}\right)$ $V^{k}=\left\{Z_{m}^{\mathfrak{k}}\right\}$ counterpart Čech and Vietoris cycles on $A$, and $\mathfrak{u}$ a finite covering of $A$. Then there exists $m(\mathfrak{U})$ such that for $m>m(\mathfrak{U}), Z_{m}^{\mathfrak{k}}$ can be projected into a cycle $\pi Z_{m}^{\mathfrak{k}}$ of $\mathfrak{U}$ (by mapping each point of $Z_{m}^{\mathfrak{k}}$ into any element of $\mathfrak{U}$ containing $i t$ ) and $\pi Z_{m}^{k} \sim Z^{k}(\mathfrak{U})$ in $\mathfrak{u}$.

The proof is obtained by using the Lebesgue number of the covering $\mathfrak{u}$ $[1$, chap. I, §5]. We omit it here:

If a compact Cech cycle $C^{k}$ in a topological space $R$ is homologous to zero on a compact subset of $R$, we shall say that $C^{k}$ is compactly homologous to zero in $R$, or bounds compactly in $R$-in symbols, $C^{k} \cong 0$ in $R$. If $R$ is metric and $V^{k}$ is a Vietoris cycle counterpart to $C^{k}$, then the compact bounding of $C^{k}$ is equivalent to the bounding of $V^{k}$, that is,

(2.2) $C^{k} \cong 0$ in $R$ is equivalent to $V^{k} \sim 0$ in $R\left({ }^{7}\right)$.

In dealing with compact Cech cycles, in order to distinguish bounding $(\sim)$

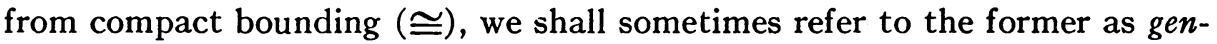
eral bounding. Unlike compact bounding, general bounding does not imply the bounding of the Vietoris counterpart. For an example, construct a $C-0$-cycle on two points of $R$. Then compact bounding of the cycle is equivalent to the two points lying together on a compact connected subset, while general bounding is equivalent merely to their lying in the same quasi-component of $R$.

However, it is still possible to state a condition on the counterpart $V^{k}$ which is equivalent to general bounding of $C^{k}$. To do this we need two preliminary notions. A Vietoris cycle $V^{k}=\left\{Z_{m}^{k}\right\}$ will be said to $\epsilon$-bound, or be $\epsilon$-homologous to zero, in $R$-in symbols, $V^{k} \sim \epsilon 0$ in $R$-if almost all the $Z_{m}^{k}$ $\epsilon$-bound in $R$ (that is, bound point chains of mesh less than $\epsilon$ ). $V^{k}$ will be said to null bound in $R$-in symbols, $V^{k} \sim_{0} 0$ in $R$-if it $\epsilon$-bounds in $R$ for all $\left.\epsilon>0{ }^{8}\right)$. Null bounding is not in itself a topological invariant. A Vietoris cycle which null bounds in $R$ under one metric may fail to do so under a different metric, even though $R$ is unchanged topologically. The property of null bounding under every metric which preserves the topology is, on the contrary, a topological invariant, and it is this property which is equivalent to general bounding. That is, we have the following theorem.

THEOREM 2. Let $R$ be a separable metric space, $C^{k}=\left\{Z^{k}(\mathfrak{U})\right\}$ a compact $C_{e c h}$ cycle in $R$, and $V^{k}=\left\{Z_{m}^{k}\right\}$ a counterpart Vietoris cycle. Then a necessary

( ${ }^{\circ}$ The cycles in the brackets are of course point cycles such that the $m$ th cycle is $\epsilon_{m}$-homologous to the $(m+1)$ th, $\epsilon_{m} \rightarrow 0$.

( ${ }^{7} V^{k} \sim 0$ of course means $V^{k}$ bounds on a compact subset.

(8) Not necessarily on a compact subset of $R$. 
and sufficient condition that $C^{k} \sim 0$ in $R$ is that $V^{k} \sim{ }_{0} 0$ in $R$ under every homeomorphic remetrization of $R\left({ }^{9}\right)$.

Proof. It will be understood without further mention of the fact that the only remetrizations we shall consider will be those which preserve topology. We first prove the necessity. Assume $C^{k} \sim 0$ and let any remetrization be imposed on $R$. By the definition of null bounding above, it is sufficient to show that for arbitrary $\epsilon>0, V^{k} \sim \epsilon 0$ in $R$. Given $\epsilon>0$, the set of neighborhoods $\{S(x, \epsilon / 3)\}$, where $x$ runs through the points of $R$, forms a covering of $R$, and by Theorem 1 , this covering has a countable star-finite refinement $\mathfrak{U}=\left\{U_{\mathfrak{i}}\right\}$. Construct a point complex $K$ isomorphic to the nerve of $\mathfrak{u}, K(\mathfrak{u})$, by choosing a point $x_{i}$ in each $U_{i}$ (the $x_{i}^{\prime}$ 's all distinct) and letting $x_{i(0)}, \cdots, x_{i(h)}$ form a simplex of $K$ if $U_{i(0)}, \cdots, U_{i(h)}$ form a simplex of $K(\mathfrak{u})$. Denote the image of $Z^{k}(\mathfrak{u})$ in $K$ by $Z^{k}$.

Since $Z^{k}(\mathfrak{U}) \sim 0$ in $\mathfrak{u}, Z^{k} \sim 0$ in $K$. But $\mathfrak{U}$ is an $\epsilon / 3$-covering, hence $K$ is a $2 \epsilon / 3$-complex. Therefore $Z^{k} \sim_{\epsilon} 0$ in $R$. We shall complete the argument by showing that $V^{k} \sim{ }_{e} Z^{k}$. Let $A$ be a compact set carrying $C^{k}$ and $V^{k}$. Only a finite number of elements of $\mathfrak{u}$ meet $A$, therefore (2.1) holds. For every $m>m(\mathfrak{U})$ we define a projection $\pi^{\prime}$ of $Z_{m}^{k}$ into $K$ as follows: Consider the projection $\pi$ of $Z_{m}^{k}$ into $\mathfrak{u}$. For each vertex $x$ of $Z_{m}^{k}$, if $\pi x=U_{i}$, we define $\pi^{\prime} x=x_{i}$. $\pi^{\prime} Z_{m}^{k}$ is obviously the image in $K$ of $\pi Z_{m}^{k}$. Therefore, since $\pi Z_{m}^{\mathfrak{k}} \sim Z^{k}(\mathfrak{U})$ in $\mathfrak{U}$, from (2.1), $\pi^{\prime} Z_{m}^{k} \sim Z^{k}$ in $K$. Hence, since $K$ is a $2 \epsilon / 3$-complex, $\pi^{\prime} Z_{m}^{k} \sim{ }_{\epsilon} Z^{k}$. Now, under $\pi^{\prime}$ each vertex was moved a distance less than $\epsilon / 3$, therefore

$$
Z_{m}^{k} \sim_{\epsilon} \pi^{\prime} Z_{m}^{k} \sim_{\epsilon} Z^{k}
$$

Since this is true for all $m>m(\mathfrak{U}), V^{k} \sim{ }_{\epsilon} Z^{k}$, which completes the proof of the necessity.

Before proving the sufficiency we demonstrate a lemma.

(2.2) If $\mathfrak{U}=\{U\}$ is a star-finite covering of a topological space and $\left\{U^{\prime}\right\}$ is a subcollection of $\{U\}$, then $U \bar{U}^{\prime}$ is a closed set.

Proof. Since each $U$ intersects only a finite number of the $\bar{U}^{\prime}, U-U \bar{U}^{\prime}$ is open. Therefore $U\left(U-U \bar{U}^{\prime}\right)$ is open. But this last is exactly the complement of $U \bar{U}^{\prime}$, which establishes the lemma.

Returning to our theorem $\left({ }^{10}\right)$, assume $C^{k}$ is not $\sim 0$ in $R$. We shall obtain a remetrization of $R$ under which $V^{k}$ is not $\sim_{0} 0$ in $R$. There exists a countable star-finite covering $\mathfrak{U}=\left\{U_{\mathfrak{i}}\right\}$ of $R$ such that $Z^{k}(\mathfrak{U})$ is not $\sim 0$ in $\mathfrak{u}$. From (1.1) we can assume that $\mathfrak{U}$ is a closure-isomorphic covering. For each $i$, let $T_{i}=\bigcup\left[U_{j} \mid U_{i} \cap U_{j}=0\right]$. From the closure-isomorphism of $\mathfrak{u}$, if $U_{i} \cap U_{j}=0$,

(9) This is a generalization of the well known property that if two points lie in the same quasi-component, then, under any metric, they can be connected by a finite sequence of points of mesh less than $\epsilon$ for all $\epsilon$, and conversely.

(10) The rest of the proof is patterned after Hurewicz [7]. 
$\bar{U}_{i} \cap \bar{U}_{j}=0 ;$ hence, from the above lemma, $\bar{U}_{i} \cap \bar{T}_{i}=0$. Therefore, since $R$ is normal, there is a continuous function $f_{i}(x)$ on $R, 0 \leqq f_{i}(x) \leqq 1$, such that

$$
f_{i}(x)=0 \quad \text { on } \quad \bar{U}_{i}, \quad f_{i}(x)=1 \quad \text { on } \bar{T}_{i} .
$$

The set of $f_{i}(x)$ 's $(i=1,2, \cdots)$ have the property that for every $x \in R$, $f_{i}(x)=1$ for almost all $i$. Hence, for any pair of points $x, y \in R, f_{i}(x)-f_{i}(y)=0$ for almost all $i$. Therefore we can define the new metric

$$
\rho_{1}(x, y)=\rho(x, y)+\sum_{i=1}^{\infty}\left|f_{i}(x)-f_{i}(y)\right|,
$$

where $\rho(x, y)$ is the given metric. It is easily verified that $\rho_{1}(x, y)$ is a homeomorphism and satisfies the axioms for a metric. Under this new metric:

$$
\text { If } \bar{U}_{i} \cap \bar{U}_{i}=0 \text { and } x \in \bar{U}_{i}, y \in \bar{U}_{j} \text {, then } \rho_{1}(x, y) \geqq 1 \text {. }
$$

$\rho_{1}$ is not the final metric. To obtain this we have to remetrize once more. Let $\left\{E_{m}\right\}$ be the collection of all finite subsets of $\left\{U_{i}\right\}$ with the following property: If $E_{m}=\left\{U_{i(j)}\right\}(j=1, \cdots, h(m))$, then:

$$
\begin{gathered}
\text { Each } \bar{U}_{i(j)} \text { intersects all the other } \bar{U}_{i(j)} \quad(j=1, \cdots, h(m)), \\
\bigcap_{j(m)} \bar{U}_{i(j)}=0 .
\end{gathered}
$$

Using the functions $f_{i}(x)$ defined in (i) we define, for each $E_{m}$, the $h(m)$ functions

$$
F_{i}^{(m)}(x)=h(m) \frac{f_{i(j)}(x)}{\sum_{0=1}^{h(m)} f_{i(o)}(x)} \quad(j=1, \cdots, h(m)) .
$$

Consider any $U_{i}$ of $\mathfrak{u}$. If $U_{i}$ does not intersect any of the $U_{i(j)}$ in $E_{m}$, then $F_{j}^{(m)}(x)=1$ on $\bar{U}_{i}(j=1, \cdots, h(m))$. But from (iv) and the star-finiteness, $U_{i}$ appears in only a finite number of the $E_{m}$ and intersects only a finite number of the others. Therefore $F_{j}^{(m)}(x)=1$ on $\bar{U}_{i}(j=1, \cdots, h(m))$ for almost all $m$. We thus have: For every $x \in R$,

$$
F_{j}^{(m)}(x)=1 \quad(j=1, \cdots, h(m)), \text { for almost all } m .
$$

It follows that given $x, y \in R, F_{j}^{(m)}(x)-F_{j}^{(m)}(y)=0(j=1, \cdots, h(m))$ for almost all $m$. Therefore we can define the new metric

$$
\rho_{2}(x, y)=\rho_{1}(x, y)+\sum_{m=1}^{\infty} \sum_{j=1}^{h(m)}\left|F_{j}^{(m)}(x)-F_{j}^{(m)}(y)\right| .
$$

$\rho_{2}$ is a homeomorphism and satisfies the axioms for a metric.

Under this new metric, if $\bigcap_{j=1}^{h} U_{i(j)}=0$, every set of points $x_{j} \in U_{i(j)}$ 
$(j=1, \cdots, h)$ is of diameter not less than 1 . To prove this we first note two properties of the functions $F_{j}^{(m)}(x)$ : At every $x \in R$ :

$$
\sum_{j=1}^{h(m)} F_{i}^{(m)}(x)=h(m)
$$

for all $m$;

$$
\text { If } x \in \bar{U}_{i(j)} \quad \text { of } \quad E_{m}, \quad F_{j}^{(m)}(x)=0 .
$$

Now assume $\bigcap_{j=1}^{h} U_{i(j)}=0$. Since $\mathfrak{u}$ is a closure-isomorphic covering, $\bigcap_{j=1}^{h} \bar{U}_{i(j)}$ $=0$ also. If two of the sets $\bar{U}_{i(j)}$ fail to intersect, we are through, from (iii). If each $\bar{U}_{i(j)}$ intersects each of the others, $\left\{U_{i(j)}\right\}(j=1, \cdots, h)$ satisfies (iv) and $(\mathrm{v})$ and therefore forms an $E_{m}$. Choose any points $x_{j} \in \bar{U}_{i(j)}(j=1, \cdots, h)$. (Now $h=h(m)$.)

$$
\rho_{2}\left(x_{1}, x_{j}\right) \geqq\left|F_{j}^{(m)}\left(x_{1}\right)-F_{j}^{(m)}\left(x_{j}\right)\right|=F_{j}^{(m)}\left(x_{1}\right)
$$

from (viii). Therefore

$$
\sum_{j=1}^{h} \rho_{2}\left(x_{1}, x_{j}\right) \geqq \sum_{j=1}^{h} F_{j}^{(m)}\left(x_{1}\right)=h
$$

from (vii). Therefore at least one of the summands, $\rho_{2}\left(x_{1}, x_{j}\right)(j=1, \cdots, h)$, is greater than or equal to 1 . The property we have just proved can be stated in the following form:

(ix) If the points $x_{1}, \cdots, x_{h}$ form a set of diameter less than 1 , and $x_{j} \in U_{i(j)}(j=1, \cdots, h)$, then $\bigcap_{j=1}^{h} U_{i(j)} \neq 0$.

We shall now show that under the metric $\rho_{2}, V^{k}$ fails to 1-bound-and therefore to null bound-in $R$. Assume $V^{k} \sim_{1} 0$. Then for almost all $m$,

$$
Z_{m}^{k} \sim_{1} 0
$$

Now for $m>m(\mathfrak{U}), Z_{m}^{\mathfrak{k}}$ projects into a cycle $\pi Z_{m}^{\mathfrak{k}}$ of $\mathfrak{U}$ such that $\pi Z_{m}^{\mathfrak{k}} \sim Z^{k}(\mathfrak{U})$ in $\mathfrak{U}$. Choose $m$ to satisfy this condition and also condition (x), and let $Y^{k+1}$ be a point chain of mesh less than 1 which is bounded by $Z_{m}^{k}$. Then from (ix), $Y^{k+1}$ can be projected into $\mathfrak{U}$, and clearly its projection is bounded by $\pi Z_{m}^{k}$. Therefore

$$
Z^{k}(\mathfrak{u}) \sim \pi Z_{m}^{k} \sim 0
$$

in $\mathfrak{u}$.

But we chose $\mathfrak{U}$ originally so that $Z^{k}(\mathfrak{U})$ is not $\sim 0$ in $\mathfrak{u}$, which gives a contradiction.

THeOREM $2^{\prime}$. Let $B$ be a closed subset (not necessarily compact) of a separable metric space $R, C^{k}$ a compact Čech cycle on $B$, and $V^{k}$ a counterpart Vietoris cycle. Then a necessary and sufficient condition that $C^{k} \sim 0$ on $B$ is that $V^{k} \sim{ }_{0} 0$ on $B$ under every homeomorphic remetrization of $R$.

This theorem follows from Theorem 2 and the isomorphism between the 
"external" and "internal" homology groups for closed subsets of $R$.

3. Neighborhood coverings. As we stated in $\$ 2$, the Cech homology groups of a closed subset $A$ of a topological space $R$ are the same whether defined by external or internal coverings. If $A$ is not closed, however, the groups obtained in these two ways are in general different. Indeed, the homology properties of $A$ given by external coverings need not be topologically invariant. For example, the open interval $0<x<1$ is homeomorphic to the circle $x^{2}+y^{2}=1$ minus the point $(0,1)$. But the 1-dimensional homology groups of the two given by external coverings in the plane are different, that of the former having the single element zero while that of the latter is the same as that of the circle. The trouble lies in the fact that the external coverings of $A$ actually give the homology properties of $\bar{A}$, and the latter set differs with different imbeddings of $A$ in $R$.

Thus, if we wish to obtain invariant homology properties of $A$ by coverings made up of open subsets of $R$, we shall have to modify our coverings. We now proceed to do this. Let $A$ be an arbitrary subset of a topological space $R$, and $G$ be any neighborhood of $A$ (that is, an open subset of $R$ containing $A$ ). Then a covering $\mathfrak{u}(G)=\{U\}$ of $G$ by open subsets $U$ of $G$ will be called a neighborhood covering of $A$. If $\mathfrak{B}(H)=\{V\}$ is another neighborhood covering of $A$ such that each $V$ is contained in some $U$, then $\mathfrak{B}(H)$ will be called a refinement of $\mathfrak{U}(G)$ and written $\mathfrak{B}(H)>\mathfrak{U}(G)$. Note that $\mathfrak{B}(H)>\mathfrak{U}(G)$ implies $H \subset G$.

Under the above definition of refinement, we can define Cech cycles on the set of all neighborhood coverings and obtain homology groups in the usual manner. Our aim in the present section is to show that the homology groups so obtained are not only topologically invariant; they are in fact the actual Cech homology groups of $A$. We do this in Theorem 3 below. First, however, we require two preliminary properties.

(3.1) Let $R$ be a completely normal (11) space, $A$ a subset of $R$, and $\mathfrak{U}=\left\{U_{i}\right\}$ a countable star-finite internal covering of $A$. Then there is a neighborhood covering of $A, \mathfrak{u}^{\prime}(G)=\left\{U_{\mathfrak{i}}\right\}$, such that $U_{\mathfrak{i}}^{\prime} \cap A=U_{\mathfrak{i}}$ and $\mathfrak{u}^{\prime}(G)$ is isomorphic to $\mathfrak{u}$ under the correspondence $U_{i} \leftrightarrow U_{i}^{\prime}\left({ }^{12}\right)$.

Proof. We shall need the following lemma of Cech's.

Lemma. If $U_{1}, \cdots, U_{h}$ are open subsets of $A$ such that $\bigcap_{j-1}^{h} U_{j}=0$, then there exist open subsets $V_{1}, \cdots, V_{h}$ of $R$ such that $V_{j} \cap A=U_{j}(j=1, \cdots, h)$ and $\bigcap_{j-1}^{n} V_{j}=0\left({ }^{13}\right)$.

(11) A space $R$ is completely normal if every two separated subsets of $R$ lie in disjoint open sets of $R$. All metric spaces are completely normal.

(2) This is an extension of a theorem of Cech for finite coverings. Cf. [3, chap. 3, \$21]. from it.

(13) Cf. [3, chap. 3, 820]. Cech's lemma has simply $V_{i} \supset U_{i}$, but our form follows easily 
We now proceed to the proof of the theorem. For each $i, U_{i}$ and $T_{i}=\cup\left[U_{j} \mid U_{i} \cap U_{j}=0\right]$ are separate. Therefore, since $R$ is completely normal, there are two disjoint open sets $M_{i}$ and $N_{i}$ of $R$ such that $M_{i} \supset U_{i}$ and $N_{i} \supset T_{i}$.

Now let $\left\{E_{m}\right\}$ be the set of all finite subsets of $\left\{U_{i}\right\}$ which have the following property: If $E_{m}=\left\{U_{i(j)}\right\}(j=1, \cdots, h(m))$, then:

(i) Each $U_{i(j)}$ intersects all the other $U_{i(j)}$ 's $(j=1, \cdots, h(m))$

$$
\bigcap_{j=1}^{h(m)} U_{i(j)}=0 .
$$

From the lemma, for each $E_{m}$, there are open sets $V_{1}^{(m)}, \cdots, V_{h(m)}^{(m)}$ of $R$ such that $V_{j}^{(m)} \cap A=U_{i(j)}(j=1, \cdots, h(m))$, and $\bigcap_{j-1}^{h(m)} V_{j}^{(m)}=0$. Consider any $U_{i}$. From (i) and the star-finiteness of $\mathfrak{U}, U_{i}$ appears in only a finite number of the $E_{m}$. Let $V_{1}, \cdots, V_{o}$ be the $V_{j}^{(m)}$ 's corresponding to it. We define

$$
U_{i}^{\prime}=M_{i} \cap\left(\cap N_{j}\right) \cap\left(\bigcap_{l=1}^{o} V_{l}\right),
$$

where $j$ runs through the natural numbers less than $i$ for which $U_{j} \cap U_{i}=0$. If we let $G=U_{i=1}^{\infty} U_{i}^{\prime}, \mathfrak{u}^{\prime}(G)=\left\{U_{i}^{\prime}\right\}$ has the required properties.

We shall call a neighborhood covering which is related to an internal covering in the above manner an elementary neighborhood covering. Although this definition is general, we shall consider only countable star-finite elementary neighborhood coverings, and we shall restrict the meaning of the word elementary to such kind. In general, to each countable star-finite internal covering there correspond many elementary neighborhood coverings, all isomorphic to each other.

(3.2) If $R$ is a separable metric space and $A$ is a subset of $R$, then every neighborhood covering of $A$ has an elementary neighborhood covering of $A$ as $a$ refinement.

Proof. Suppose $\mathfrak{u}(G)$ is a neighborhood covering of $A$. Since $R$ is separable metric, we can assume $\mathfrak{u}(G)$ is countable star-finite and write it $\mathfrak{u}(G)=\left\{U_{i}\right\}$. Let $\left\{U_{i(j)}\right\}$ be the set of all $U_{i}^{\prime}$ 's which intersect $A$, and set $V_{j}=U_{i(j)} \cap A$. Then $\mathfrak{B}=\left\{V_{j}\right\}$ is a countable star-finite internal covering of $A$. Let $\mathfrak{B}^{\prime}(H)=\left\{V_{j}^{\prime}\right\}$ be an elementary neighborhood covering obtained from $\mathfrak{B}$ by (3.1) with the additional condition that $V_{j}^{\prime} \subset U_{i(j)}$ for all $j$. Then $\mathfrak{B}^{\prime}(H)$ is the required refinement of $\mathfrak{u}(G)$.

We now prove the principal theorem of this section.

THEOREM 3. If $R$ is a separable metric space (14) and $A$ is an arbitrary subset of $R$, then the Cech homology groups determined by neighborhood coverings of $A$ are isomorphic to those given by internal coverings.

(14) All we need is that $R$ be completely normal and the countable star-finite coverings form a complete family for all subsets. 
Proof. All the coverings in this proof will be countable star-finite. From (3.2) the elementary neighborhood coverings form a complete family. We shall therefore restrict ourselves to them and omit the adjective elementary. Let $C^{\prime k}=\left\{Z^{k}\left(\mathfrak{u}^{\prime}(G)\right)\right\}$ be a $C$-k-cycle defined on the neighborhood coverings of $A$. For each internal covering $\mathfrak{u}$, choose any neighborhood covering $\mathfrak{u}^{\prime}(G)$ corresponding to it (that is, related to it as in (3.1)), and let $Z^{k}(\mathfrak{U})$ be the image of $Z^{k}\left(\mathfrak{U}^{\prime}(G)\right)$. Now suppose that $\mathfrak{B}>\mathfrak{U}, \mathfrak{B}^{\prime}(H)$ is a neighborhood covering corresponding to $\mathfrak{B}$ such that $\mathfrak{B}^{\prime}(H)>\mathfrak{u}^{\prime}(G)$, and $Z^{k}(\mathfrak{B})$ is the image of $Z^{k}\left(\mathfrak{B}^{\prime}(H)\right)$. If $\pi$ is any projection from $\mathfrak{B}$ to $\mathfrak{U}$ and $\pi^{\prime}$ the corresponding projection from $\mathfrak{B}^{\prime}(H)$ to $\mathfrak{U}^{\prime}(G)$, it follows from $\pi^{\prime} Z^{k}\left(\mathfrak{B}^{\prime}(H)\right) \sim Z^{k}\left(\mathfrak{U}^{\prime}(G)\right)$ in $\mathfrak{u}^{\prime}(G)$ that

$$
\pi Z^{k}(\mathfrak{B}) \sim Z^{k}(\mathfrak{U})
$$

in $\mathfrak{u}$.

If we should pick a different neighborhood covering $\mathfrak{U}_{\mathfrak{1}}^{\prime}\left(G_{1}\right)$ corresponding to $\mathfrak{u}$, we might get a different cycle $Z_{\mathfrak{l}}^{\mathfrak{k}}(\mathfrak{U})$ in $\mathfrak{U}$. But we need only choose $\mathfrak{B}^{\prime}(H)$ to be a refinement of both $\mathfrak{u}^{\prime}(G)$ and $\mathfrak{U}_{\mathfrak{1}}^{\prime}\left(G_{1}\right)$, and then from (i) we would have $Z_{\mathfrak{1}}^{\mathfrak{k}}(\mathfrak{U}) \sim Z^{k}(\mathfrak{U})$ in $\mathfrak{u}$. It follows that $Z^{k}(\mathfrak{U})$ is uniquely determined up to a homology. It also follows from (i) that $\left\{Z^{k}(\mathfrak{U})\right\}$ is a Cech cycle $C^{k}$.

Since $Z^{k}(\mathfrak{U}) \sim 0$ in $\mathfrak{U}$ if and only if $Z^{k}\left(\mathfrak{U}^{\prime}(G)\right) \sim 0$ in $\mathfrak{u}^{\prime}(G)$, we have that $C^{k} \sim 0$ if and only if $C^{\prime k} \sim 0$. We thus have defined an isomorphism of the "neighborhood" Cech- $k$-homology group into the "internal" one. To complete the proof, we shall show that every "internal" Cech- $k$-cycle corresponds to a "neighborhood" one. Let $C^{k}=\left\{Z^{k}(\mathfrak{u})\right\}$ be a $C$-k-cycle defined on the internal coverings. For each neighborhood covering $\mathfrak{u}^{\prime}(G)$, let $\mathfrak{u}$ be the internal covering corresponding to it and let $Z^{k}\left(\mathfrak{U}^{\prime}(G)\right)$ be the image of $Z^{k}(\mathfrak{U})$. Suppose $\mathfrak{B}^{\prime}(H)>\mathfrak{U}^{\prime}(G), \pi^{\prime}$ is a projection from $\mathfrak{B}^{\prime}(H)$ into $\mathfrak{U}^{\prime}(G)$, and $\pi$ is the corresponding projection from $\mathfrak{B}$ into $\mathfrak{U}$. Then since $\pi Z^{k}(\mathfrak{B}) \sim Z^{k}(\mathfrak{U})$ in $\mathfrak{U}$, it follows that $\pi^{\prime} Z^{k}\left(\mathfrak{B}^{\prime}(H)\right) \sim Z^{k}\left(\mathfrak{U}^{\prime}(G)\right)$ in $\mathfrak{u}^{\prime}(G)$. Therefore $\left\{Z^{k}\left(\mathfrak{U}^{\prime}(G)\right)\right\}$ is a Cech cycle, and clearly it goes into $C^{k}$ under our isomorphism. This completes the proof.

4. Polyhedral complexes. By a polyhedral complex $K$, we shall mean a star-finite polyhedral complex in the classical sense, with possibly an infinite number of simplexes. A subdivision of $K$ will, in this paper, always mean a simplicial subdivision, and we shall omit the adjective "simplicial" hereafter. We shall denote subdivisions of $K$ by the small German letters $\mathfrak{u}, \mathfrak{b}, \mathfrak{w}$, and their vertices by the small italic letters $u_{i}, v_{i}, w_{i}$. Given a subdivision $\mathfrak{u}$ of $K$ with the vertices $\left\{u_{i}\right\}$, we shall denote the star of each $u_{i}$ by $U_{i}$. Then $\mathfrak{u}=\left\{U_{i}\right\}$ is a countable star-finite covering of $K$ isomorphic to $\mathfrak{u}$. We shall call it the subdivision covering derived from $\mathfrak{u}$.

(4.1) If $K$ is a polyhedral complex, the subdivision coverings form a complete family for $K$. In fact, if $\mathfrak{u}_{0}$ is a given subdivision, we may limit ourselves to the subdivision coverings derived from subdivisions of $\mathfrak{u}_{0}$.

Proof. If $K$ is finite, it is compact. Therefore every covering of $K$ has a 
finite refinement. This has a Lebesgue number $\eta$, and by repeated barycentric subdivision of $\mathfrak{u}_{0}$, we can obtain a subdivision of mesh less than $\eta / 3$. Then the covering derived from this subdivision is the required refinement.

Suppose $K$ is infinite. Then it is the union of a sequence $\left\{K_{m}\right\}$ of finite subcomplexes of $\mathfrak{u}_{0}$ such that each $K_{m}$ meets only $K_{m-1}$ and $K_{m+1}$. Now given any covering $\mathfrak{u}$ of $K$, we proceed inductively. Since $K_{1}$ is finite, we can subdivide it until we get a subdivision whose derived covering is a refinement of $\mathfrak{U}$ on $K_{1}$. Extend this subdivision to those simplexes of $K_{2}$ whose faces meet $K_{1}$. Assume that we have a subdivision of $\bigcup_{j=1}^{m-1} K_{j}$ whose derived covering is a refinement of $\mathfrak{U}$ and that this subdivision has been extended to those simplexes of $K_{m}$ whose faces meet $K_{m-1}$. Then subdivide $K_{m}$ in the same manner and extend the subdivision to those simplexes of $K_{m-1}$ and $K_{m+1}$ whose faces meet $K_{m}$. In this way each $K_{m}$ has its final subdivision after the $(m+1)$ th stage, and we thus obtain a well defined subdivision of $\mathfrak{u}_{0}$ which satisfies the theorem.

By the $k$-dimensional homology group of a subdivision $\mathfrak{u}$, we shall mean the homology group obtained by using simplexes of $\mathfrak{u}$. In this paper we shall consider only finite chains and cycles of subdivisions.

We now show the equivalence of the Cech theory with the classical theory for polyhedral complexes.

THEOREM 4. If $K$ is a polyhedral complex and $\mathfrak{u}_{0}$ is any subdivision of $K$, the $k$-dimensional Cech homology group of $K$, the k-dimensional Vietoris homology group of $K$, and the $k$-dimensional homology group of $\mathfrak{u}_{0}$ are all isomorphic, for all $k$.

Proof. The isomorphism of the Cech- $k$-homology group of $K$ and the homology group of $\mathfrak{u}_{0}$ follows directly from (4.1) and the fact that every subdivision of $\mathfrak{u}_{0}$ has the same $k$-homology group as $\mathfrak{u}_{0}$.

Now every cycle of $\mathfrak{u}_{0}$ is in a finite subcomplex of $\mathfrak{u}_{0}$, and if it bounds, bounds a chain of a finite subcomplex of $\mathfrak{u}_{0}$. Therefore, since finite complexes are compact, every Cech cycle is homologous to a compact Cech cycle, and if it bounds, bounds compactly. Hence the Cech- $k$-homology group obtained by using compact cycles and compact bounding is also isomorphic to the $k$-homology group of $\mathfrak{u}_{0}$. But this last group is the same as the Vietoris- $k$ homology group, which completes the proof.

CoROLlary. The general k-dimensional Cech homology group of a polyhedral complex is isomorphic to the homology group obtained by using compact cycles and compact bounding.

We shall denote a $k$-dimensional singular cycle( $\left.{ }^{16}\right)$ of $K$ by $z^{k}$. A cycle of any subdivision $\mathfrak{u}$ of $K$ is, of course, also a singular cycle. We shall denote it by $z^{k}(\mathfrak{u})$. In what follows we shall have need of two theorems which are proved

(अ) C. [2, chap. VIII, $\$ 5$ and chap. IX, $\$ \$ 1$ and 2]. 
by the well known fundamental deformation process for polyhedral complexes $\left({ }^{15}\right)$. We state them here without proof.

(4.2) Let $z^{k}$ be a singular cycle of a polyhedral complex $K$. If $\mathfrak{u}$ is any subdivision of $K$, there is a cycle $z^{k}(\mathfrak{u})$ of $\mathfrak{u}$ such that $z^{k} \sim z^{k}(\mathfrak{u})$ in $K\left({ }^{10}\right)$. Further, if $z^{k} \sim 0$ in $K\left({ }^{16}\right), z^{k}(\mathfrak{u}) \sim 0$ in $\mathfrak{u}$, and conversely.

COROLLARY. The k-dimensional homology group given by singular cycles of $K$ is isomorphic to the k-dimensional homology group of any subdivision of $K$.

Before stating the second theorem, we make some preliminary remarks. An open subset of a polyhedral complex is itself a polyhedral complex. Let $\mathfrak{u}$ be a subdivision of $K$ and $\mathfrak{u}$ its derived subdivision covering $\left({ }^{17}\right)$. If $G$ is an open subset of $K$, we can apply (4.1) to $G$ and obtain a subdivision $\mathfrak{v}$ of $G$ whose derived subdivision covering $\mathfrak{B}$ is a refinement of $\mathfrak{U}$ in $G$. We shall denote this by $\mathfrak{B}>\mathfrak{U}$.

(4.3) Given an open subset $G$ of a polyhedral complex $K$, let $\mathfrak{u}$ be a subdivision of $K, \mathfrak{v}$ a subdivision of $G$, and $\mathfrak{U}$ and $\mathfrak{B}$ their derived subdivision coverings, with $\mathfrak{B}>\mathfrak{U}$. Suppose $z^{k}(\mathfrak{b})$ and $\mathfrak{z}^{k}(\mathfrak{u})$ are cycles of $\mathfrak{v}$ and $\mathfrak{u}$ respectively, and denote their images in $\mathfrak{B}$ and $\mathfrak{U}$ by $Z^{k}(\mathfrak{B})$ and $Z^{k}(\mathfrak{U})$. Then, if $\pi$ is a projection from $\mathfrak{B}$ into $\mathfrak{U}$, a necessary and sufficient condition that $\pi Z^{k}(\mathfrak{B}) \sim Z^{k}(\mathfrak{U})$ in $\mathfrak{U}$ is that $z^{k}(\mathfrak{b}) \sim z^{k}(\mathfrak{u})$ in $K$.

Now let $A$ be an arbitrary subset of $K$. A geometric Cech-k-cycle of $A$ will be defined as a collection $C^{k}=\left\{z^{k}(G)\right\}$, where $G$ runs through all the neighborhoods of $A, z^{k}(G)$ is a singular cycle in $G$, and for every neighborhood $H$ of $A$ such that $H \subset G, z^{k}(H) \sim z^{k}(G)$ in $G$. If $z^{k}(G) \sim 0$ in $G$ for all $G$, we shall say that $C^{k}$ bounds in $A$ or $C^{k} \sim 0$ in $A$. The geometric Cech cycles define homology groups in the usual manner. Again, these homology groups are not only topological invariants of $A$; they are the actual Cech homology groups of $A$. That is, we have the following theorem.

(4.4) If $A$ is an arbitrary subset of a polyhedral complex, the $k$-dimensional homology group defined by the geometric Cech-k-cycles of $A$ is isomorphic to the Cech-k-homology group of $A$, for all $k$.

Proof. From Theorem 3, we need only consider neighborhood coverings of $A$, and from (4.1) we may confine ourselves to those derived from subdivisions of the neighborhoods. Hence we shall assume in this proof that all our coverings are of the latter sort. Consider a Cech-k-cycle $C^{k}=\left\{Z^{k}(\mathfrak{U}(G))\right\}$ of $A$. Given any $\mathfrak{u}(G)$, denote the subdivision from which it was derived by $\mathfrak{u}(G)$ and the image $\left.{ }^{18}\right)$ of $Z^{k}(\mathfrak{U}(G))$ there by $z^{k}(\mathfrak{u}(G))$. If $\mathfrak{B}(H)>\mathfrak{U}(G)$ and $\pi$ is a projection from $\mathfrak{B}(H)$ into $\mathfrak{u}(G)$, it follows from $\pi Z^{k}(\mathfrak{B}(H)) \sim Z^{k}(\mathfrak{U}(G)$ ) in $\mathfrak{U}(G)$ and from (4.3), that

(16) That is, their difference bounds a singular chain.

(17) See the opening paragraph of this section.

(18) Under the isomorphism of $\mathfrak{U}$ and $\mathfrak{u}$. 


$$
z^{k}(\mathfrak{b}(H)) \sim z^{k}(\mathfrak{u}(G))
$$

in $G$.

Now we make correspond to $C^{k}$ a geometric Cech-k-cycle $C_{1}^{k}=\left\{z^{k}(G)\right\}$ in the following manner: For each neighborhood $G$ of $A$, choose any $\mathfrak{u}(G)$ and let $z^{k}(\mathfrak{u}(G))$ be $z^{k}(G)$. By letting $H=G$ in (i), it is readily shown that $z^{k}(G)$ is uniquely determined up to a homology. It also follows from (i) that $\left\{z^{k}(G)\right\}$, where $G$ runs through all the neighborhoods of $A$, is a geometric Čech-k-cycle. We denote it by $C_{1}^{k}$. Since $z^{k}(G) \sim 0$ in $G$ if and only if $Z^{k}(\mathfrak{U}(G)) \sim 0$ in $\mathfrak{U}(G)$, we have that $C_{\mathfrak{l}}^{\mathfrak{k}} \sim 0$ if and only if $C^{k} \sim 0$. We thus have defined an isomorphism of the Cech- $k$-homology group of $A$ into the $k$-dimensional homology group given by the geometric Cech- $k$-cycles. To complete the proof, we shall show that to every geometric Cech- $k$-cycle there corresponds a Cech-k-cycle. Let $C^{k}=\left\{z^{k}(G)\right\}$ be a geometric Cech-k-cycle. For every neighborhood $G$ and subdivision $\mathfrak{u}(G)$ of $G$, choose $z^{k}(\mathfrak{u}(G)) \sim z^{k}(G)$ by (4.2), and let $Z^{k}(\mathfrak{u}(G))$ be the image of $z^{k}(\mathfrak{u}(G))$ in the derived covering $\mathfrak{u}(G)$. Then $\left\{Z^{k}(\mathfrak{U}(G))\right\}$ is a Cech-k-cycle $C^{k}$. For $\mathfrak{B}(H)>\mathfrak{U}(G)$ implies that

$$
z^{k}(\mathfrak{v}(H)) \sim z^{k}(H) \sim z^{k}(G) \sim z^{k}(\mathfrak{u}(G))
$$

in $G$;

hence, from (4.3), $\pi Z^{k}(\mathfrak{B}(H)) \sim Z^{k}(\mathfrak{U}(G))$ in $G$. It is easily seen that $C^{k}$ maps into a geometric Cech-k-cydle which is homologous to $C_{1}^{k}$. This completes the proof.

THEOREM 5. If $A$ is an arbitrary subset of a polyhedral complex $K$, and $C^{k}$ is a compact Cech cycle in $A$, then a necessary and sufficient condition that $C^{k} \sim 0$ in $A$ is that $C^{k} \cong 0$ in every neighborhood of $A$.

Theorem 5 follows from the more general Theorem $5^{\prime}$ which we prove below. First we shall demonstrate a lemma. In what follows, we shall use the single word independent to mean linearly independent relative to homologies.

(4.5) If the Cech cycles $C_{\mathfrak{k}}^{\mathbf{k}}=\left\{Z_{\mathfrak{k}}^{\mathbf{k}}(\mathfrak{U})\right\}(i=1, \cdots, h)$ in a topological space $R$ are independent there, then there is a covering $\mathfrak{U}$ such that $Z_{\mathfrak{i}}^{\mathfrak{k}}(\mathfrak{u})(i=1, \cdots, h)$ are independent in $\mathfrak{U}$.

Proof. Given a Cech cycle $C^{k}=\left\{Z^{k}(\mathfrak{u})\right\}$ and a covering $\mathfrak{u}$, if $Z^{k}(\mathfrak{u}) \sim 0$ in $\mathfrak{U}$, we shall write $C^{k} \sim 0$ in $\mathfrak{U}$. Assume there is no covering satisfying the theorem. Then for each covering $\mathfrak{U}$, there is a linear combination $C^{k}(\mathfrak{U})$ $=\sum_{\mathfrak{l}=1}^{h} a_{\mathfrak{i}}(\mathfrak{U}) C_{\mathfrak{k}}^{\mathfrak{k}}$ which is $\sim 0$ in $\mathfrak{U}$. Choose any covering $\mathfrak{U}_{0}$. Since the $C_{\mathfrak{k}}^{\mathfrak{k}}$ $(i=1, \cdots, h)$ are independent, there is a $\mathfrak{U}_{1}>\mathfrak{U}_{0}$ such that $C^{k}\left(\mathfrak{U}_{0}\right)$ is not $\sim 0$ in $\mathfrak{U}_{1}$. Similarly, there is a $\mathfrak{U}_{2}>\mathfrak{U}_{1}$ such that $C^{k}\left(\mathfrak{U}_{1}\right)$ is not $\sim 0$ in $\mathfrak{U}_{2}$. Proceeding, we obtain $h+1$ linear combinations $C^{k}\left(U_{0}\right), \cdots, C^{k}\left(U_{h}\right)$ such that

$$
\begin{aligned}
\mathfrak{U}_{h}>\mathfrak{U}_{h-1}>\cdots>\mathfrak{U}_{0}, & \\
C^{k}\left(\mathfrak{U}_{j}\right) \sim 0 \text { in } \mathfrak{U}_{j}, & j=0, \cdots, h, \\
C^{k}\left(\mathfrak{U}_{j}\right) \text { is not } \sim 0 \text { in } \mathfrak{U}_{j+1}, & j=0, \cdots, h-1 .
\end{aligned}
$$


Since we have $h+1$ linear forms in $h$ quantities, one of the linear forms must be a linear combination of the rest $\left({ }^{19}\right)$. Let $C^{k}\left(\mathfrak{U}_{j}\right)$ be the first such. Then

$$
C^{k}\left(\mathfrak{U}_{j}\right)=b_{j+1} C^{k}\left(\mathfrak{U}_{j+1}\right)+\cdots+b_{h} C^{k}\left(\mathfrak{U}_{h}\right) .
$$

Since each term on the right bounds in a refinement of $\mathfrak{U}_{j+1}$, it bounds in $\mathfrak{U}_{j+1}$. Hence $C^{k}\left(\mathfrak{U}_{j}\right)$ bounds in $\mathfrak{u}_{j+1}$. But $\mathfrak{U}_{j+1}$ was picked so that $C^{k}\left(\mathfrak{U}_{j}\right)$ is not $\sim 0$ there, which gives a contradiction.

TheOREM $5^{\prime}$. If $A$ is an arbitrary subset of a polyhedral complex $K$ and $C_{i}^{k}$ $(i=1, \cdots, h)$ are compact $C$ ech cycles in $A$, then a necessary and sufficient condition that they be independent relative to general homologies in $A$ is that there exist a neighborhood $G$ of $A$ in which they are independent relative to compact homologies.

Proof. Assume $C_{i}^{\mathbf{k}}(i=1, \cdots, h)$ are not independent in $A$ relative to general homologies, that is, some linear combination of them, $C^{k}=\sum_{i-1}^{k} a_{i} C_{t}^{k}$, is $\sim 0$ in $A$. Consider any neighborhood $G$ of $A$. Since $C^{k} \sim 0$ in every neighborhood covering of $A$, it is $\sim 0$ in every covering of $G$. Therefore, from the corollary of Theorem $4, C^{k} \cong 0$ in $G$.

Now assume $C_{i}^{k}(i=1, \cdots, h)$ are independent in $A$. Then, from (4.5) above, there is a covering $\mathfrak{u}$ of $A$ in which they are independent. The neighborhood coverings form a complete family, hence we may assume $\mathfrak{U}$ is actually a neighborhood covering $\mathfrak{u}(G)$. Since $\mathfrak{u}(G)$ is a covering of $G$, this means the $C_{i}^{k}(i=1, \cdots, h)$ are independent in $G$ relative to general homologies (hence, of course, independent relative to compact homologies there).

Corollary 1. Let $A$ be an arbitrary subset of a polyhedral complex $K$, $C_{i}^{\mathbf{k}}(i=1, \cdots, h)$ be compact $C_{\text {ech }}$ cycles in $A$, and $V_{i}^{\boldsymbol{k}}$ be a counterpart Vietoris cycle to $C_{\mathbf{k}}^{\mathbf{k}}(i=1, \cdots, h)$. Then a necessary and sufficient condition that the $C_{\boldsymbol{i}}^{\mathbf{k}}$ be independent relative to general homologies in $A$ is that there exist a neighborhood $G$ of $A$ in which the $V_{\mathbb{k}}^{k}$ are independent $\left({ }^{20}\right)$.

Corollary 2. If $A$ is an arbitrary subset of a polyhedral complex $K$ and $C^{k}$ and $V^{k}$ are counterpart compact $\nearrow^{\top}$ ech and Vietoris cycles in $A$, then a necessary and sufficient condition that $C^{k} \sim 0$ in $A$ is that $V^{k} \sim 0$ in every neighborhood of $A\left({ }^{20}\right)$.

5. Duality theorems. Let $F$ be a closed subset of the $n$-sphere $S^{n}$. As is well known, for a Vietoris cycle $V^{n-k-1}$ of $F$ and a singular cycle $z^{k}$ of $S^{n}-F$ a linking number $\nu\left(z^{k}, V^{n-k-1}\right)$ may be defined( $\left.{ }^{21}\right)$. The linking number can be defined in the same manner between $z^{k}$ and a Cech cycle $C^{n-k-1}$
(19) We recall that our coefficients are in a field.
(20) Cf, footnote 7.
(21) Cf., for instance, [1, chap. 4]. 
$=\left\{z^{n-k-1}(G)\right\}$ on $F$ ( $G$ running through all the neighborhoods of $F$ ). We let $G_{0}$ be a neighborhood of $F$ not meeting $z^{k}$ and restrict $G$ to neighborhoods contained in $G_{0}$. Then $\nu\left(z^{k}, z^{n-k-1}(G)\right)$ is defined and is clearly independent of $G$. We can therefore take it as our $\nu\left(z^{k}, C^{n-k-1}\right)$. It is easily shown that if $z_{1}^{k} \sim z^{k}$ outside of $F$,

$$
\nu\left(z_{1}^{k}, C^{n-k-1}\right)=\nu\left(z^{k}, C^{n-k-1}\right) .
$$

We note, also, that if $V^{n-k-1}$ is a Vietoris counterpart of $C^{n-k-1}$,

$$
\nu\left(z^{k}, C^{n-k-1}\right)=\nu\left(z^{k}, V^{n-k-1}\right) .
$$

Now suppose $A$ is an arbitrary subset of $S^{n}$. We can extend our definition of linking number to one between a general Cech cycle of $A$ and a compact Cech cycle of $S^{n}-A$. Suppose $C^{k}=\left\{z^{k}(G)\right\}$ is a general Cech cycle of $A$ and $C^{n-k-1}$ a compact Cech cycle in $S^{n}-A$. Let $F$ be a compact subset of $S^{n}-A$ containing $C^{n-k-1}$, and restrict all the neighborhoods $G$ of $A$ to lie in $S^{n}-F$. Then $\nu\left(z^{k}(G), C^{n-k-1}\right)$ is defined, as we have shown above, and since for $H \subset G$, $z^{k}(H) \sim z^{k}(G)$ in $G$, this linking number is independent of $G$. We can therefore take it as our $\nu\left(C^{k}, C^{n-k-1}\right)$. If both $C^{k}$ and $C^{n-k-1}$ are compact, we have the following immediate theorem.

(5.3) If $C^{k}$ and $C^{n-k-1}$ are compact Čech cycles in disjoint subsets of $S^{n}$, and $V^{k}$ and $V^{n-k-1}$ are their respective Vietoris counterparts, then

$$
\nu\left(C^{\dot{k}}, C^{n-k-1}\right)=\nu\left(V^{k}, V^{n-k-1}\right)\left({ }^{21}\right) .
$$

We shall now prove a generalization of the Alexander duality theorem to arbitrary subsets of the $n$-sphere $S^{n}$. We first demonstrate a lemma.

(5.4) Lemma. Let $A$ be an arbitrary subset of $S^{n}$ and $C_{i}^{\mathbf{k}}(i=1, \cdots, h)$ be compact Cech cycles in $A$. If the $C_{i}^{\boldsymbol{k}}(i=1, \cdots, h)$ are independent relative to compact homologies in some neighborhood of $A$, then $S^{n}-A$ contains compact Cech cycles $C_{i}^{n-k-1}(i=1, \cdots, h)$ which are independent relative to compact homologies in some neighborhood of $S^{n}-A$. The latter cycles can be chosen so that

$$
\nu\left(C_{i}^{k}, C_{j}^{n-k-1}\right)=\delta_{i j} \quad(i, j=1, \cdots, h),
$$

where $\delta_{i j}$ is the Kronecker $\delta$.

Proof. Let $F$ be a compact subset of $A$ containing the $C_{i}^{k}(i=1, \cdots, h)$, and let them be independent relative to compact homologies in the neighborhood $G$ of $A$. Then by the duality for closed sets and (5.3) above, $S^{n}-G$, which is a closed set, contains $C_{i}^{n-k-1}(i=1, \cdots, h)$ such that

$$
\nu\left(C_{i}^{k}, C_{j}^{n-k-1}\right)=\delta_{i j} \quad(i, j=1, \cdots, h) .
$$

Because of (i), the $C_{\mathfrak{t}}^{n-\mathbf{k - 1}}$ are independent relative to compact homologies in 
$S^{n}-F$. But $S^{n}-F$ is an open set containing $S^{n}-A$, therefore a neighborhood of $S^{n}-A$, which completes the proof.

THEOREM 6. A necessary and sufficient condition that an arbitrary subset $A$ of $S^{n}$ contain $h$ compact Čech-k-cycles which are independent relative to general homologies in $A$ is that $S^{n}-A$ contain $h$ compact $\nearrow_{\text {ech }}(n-k-1)$-cycles which are independent relative to general homologies in $S^{n}-A$.

Proof. Since $A$ is arbitrary, the theorem is symmetric in $A$ and $S^{n}-A$. Hence we need only prove the necessity. This follows directly from Theorem $5^{\prime}$ and (5.4) above.

If we remove the condition that a space $R$ be compact, a multiplicity of homology properties may immediately be defined which do not appear, or rather are not distinct, in the compact case. Perhaps the strongest of these is that $R$ contain a compact Cech cycle which fails to bound generally. This is the property we assumed on $A$ in Theorem 6 above-we are now stating it in terms of a single cycle for the sake of clarity-and, as we saw there, it implies the same property for $S^{n}-A$. We list five additional properties below, three obtained by weakening the conditions on the cycle and two by weakening the conditions on the non-bounding. (We could list others, but these will suffice for our purpose.) As we shall show, varying the conditions on the cycle in a subset of $S^{n}$ seems to be dual to varying the conditions on the bounding in the complement. We therefore list the properties obtained by the former process as properties of $A$ and those obtained by the latter as properties of $S^{n}-A$. This is simply for our discussion of duality; all the properties can of course be defined for any topological space. We also list the above strong property of Theorem 6 in both $A$ and $S^{n}-A$ so that the other properties may be compared with it.

\section{$A$}

I. There is a compact $C^{k}$ in $A$ not $\sim 0$ in $A$.

II. There is a general $C^{k}$ in $A$ not $\sim 0$ in $A$.

III. There is a covering of $A$ every refinement of which has an essential ( $\left.{ }^{22}\right)$ non-bounding $k$-cycle.

IV. There is a covering $\mathfrak{U}$ of $R$ every refinement of which has a $k$-cycle which projects into a non-bounding cycle of $\mathfrak{u}$.

$$
S^{n}-A
$$

$I^{\prime}$. There is a compact $C^{n-k-1}$ in $S^{n}-A$ not $\sim 0$ in $S^{n}-A$.

III'. There is a compact $C^{n-k-1}$ in $S^{n}-A$ not $\cong 0$ in $S^{n}-A$.

IV'. There is a compact subset $F_{0}$ of $S^{n}-A$ such that for every compact subset $F$ of $A$ containing $F_{0}$, some $C^{n-k-1}$ of $F_{0}$ is not $\cong 0$ in $F$.

(2) A cycle $Z(\mathfrak{u})$ of a covering $\mathfrak{u}$ is essential if every refinement of $\mathfrak{u}$ has a cycle which projects into a cycle of $\mathfrak{u}$ which is $\sim Z(\mathfrak{U})$. 
Note that in each column, each property is weaker than the one above. Also, for a compact space, if the dimensions in the two columns were the same, all six properties would reduce to the same one.

We showed in Theorem 6 that $I$ and $I^{\prime}$ are dual, that is, each implies the other. Properties IV and IV' are also dual. Before showing this, we make a definition. If a space $R$ has property IV we shall say it is $k$-disconnected; otherwise it will be called $k$-connected.

THEOREM 7. Let $A$ be an arbitrary subset of $S^{n}$. A necessary and sufficient condition that $A$ be $k$-disconnected is that $S^{n}-A$ contain a compact subset $F_{0}$ such that for every compact subset $F$ of $S^{n}-A$ containing $F_{0}$, some Cech$(n-k-1)$-cycle of $F_{0}$ fails to bound in $F$.

Proof. Assume $A$ is $k$-disconnected. In terms of neighborhoods and singular cycles this is equivalent to the following statement: There is a neighborhood $G$ of $A$ such that every neighborhood $H$ of $A, H \subset G$, has a $z^{k}(H)$ not $\sim 0$ in $G$. Then $F_{0}=S^{n}-G$ has the required properties. For assume $F_{0} \subset F \subset S^{n}-A$, $F$ compact. $S^{n}-F$ is a neighborhood $H$ of $A$ and it is contained in $G$. Hence it contains a $z^{k}(H)$ not $\sim 0$ in $G$. Therefore, by the duality for closed sets, $F_{0}$ contains a $C^{n-k-1}$ not $\cong 0$ in $F$.

Conversely, assume we are given $F_{0}$ in $S^{n}-A$ satisfying the hypothesis. Let $G=S^{n}-F_{0}$. Then if $H$ is a neighborhood of $A, H \subset G, F_{0}$ contains a $C^{n-k-1}$ not $\cong 0$ in $S^{n}-H$. Hence $H$ contains a $z^{k}(H)$ not $\sim 0$ in $G$. This completes the proof.

COROLlaRy. If $A$ is an arbitrary subset of $S^{n}$, and $S^{n}-A$ contains a compact Cech-(n-k-1)-cycle which fails to bound compactly there, then $A$ is $k$-disconnected.

This follows from the fact that property III' implies property IV'.

Concerning the other properties in our list, we can show that property III implies property III':

THEOREM 8. Let $A$ be an arbitrary subset of $S^{n}$. If there is a covering of $A$ every refinement of which has an essential nonbounding $k$-cycle, then $S^{n}-A$ has a compact Cech-(n-k-1)-cycle which fails to bound compactly in $S^{n}-A$.

Proof. In terms of neighborhoods and singular cycles, the hypothesis on $A$ implies the following property: There is a neighborhood $G$ of $A$ with a singular cycle $z^{k}(G)$ not $\sim 0$ in $G$ such that if $H$ is a neighborhood of $A, H \subset G$, there is a $z^{k}(H) \sim z^{k}(G)$ in $G$. By the duality for closed sets, $F_{0}=S^{n}-G$ contains a $C^{n-k-1}$ linked with $z^{k}(G)$. Then $C^{n-k-1}$ is not $\cong 0$ in $S^{n}-A$. For assume it is, that is, $F_{0} \subset F \subset S^{n}-A, F$ compact, such that $C^{n-k-1}$ bounds in $F . S^{n}-F$ is a neighborhood $H$ of $A$ contained in $G$. Hence there is a $z^{k}(H) \sim z^{k}(G)$ in $G$. Therefore $C^{n-k-1}$ is linked with $z^{k}(H)$, contradicting the assumption that it bounds in $F$. 
COROLlaRy. If an arbitrary subset $A$ of $S^{n}$ contains a general Cech-k-cycle which fails to bound in $A, S^{n}-A$ contains a compact Čech-(n-k-1)-cycle which fails to bound compactly in $S^{n}-A$.

This follows from the fact that property II implies property III.

The converse of Theorem 8 is not true, as is shown by the following example: In the Cartesian plane, let $M$ be the curve $y=\sin 1 / x, 0<x<2 / 3 \pi$, and $N$ an arc from $(0,-1)$ to $(2 / 3 \pi,-1)$ not meeting $M$ and, except for its end point, lying entirely in the fourth quadrant. Then, if $A=M \cup N$, the complement of $A$ has compact Cech-0-cycles which fail to bound there relative to compact homologies, and yet given any covering $\mathfrak{U}$ of $A$, there are refinements of $\mathfrak{U}$ which have no essential 1-cycles. (Hence, of course, $A$ has no non-bounding Cech-1-cycle. It is, however, 1-disconnected, as can be seen from the corollary of Theorem 7.)

The blank space opposite property II is significant. We have had considerable difficulty in finding conditions on $S^{n}-A$ (short of condition $\mathrm{I}^{\prime}$ ) which will imply the existence of (non-bounding) Cech cycles in $A$. The problem seems to be closely linked with an important general question:

Can there actually exist, in a separable metric space, Cech cycles which are not compact or homologous to compact cycles?

We do not know the answer to this question.

Before leaving the discussion of our list of properties, we demonstrate the remarkable fact that for the dimension $n-1$, properties I' $^{\prime}$ and III' are equivalent, that is, general bounding is equivalent to compact bounding.

TheOREM 9. Let $B$ be an arbitrary subset of $S^{n}$ and $C^{n-1}$ a compact Čech$(n-1)$-cycle of $B$. Then if $C^{n-1} \sim 0$ in $B$, it is $\cong 0$ in $B\left({ }^{23}\right)$.

Proof. Let $F_{0}$ be a compact subset of $B$ containing $C^{n-1}$, and assume $C^{n-1}$ is not $\cong 0$ in $B$. Let $F$ be the compact subset of $B$ obtained by adding to $F_{0}$ all the components of its complement containing no point of $S^{n}-B$. From the remaining components, choose a set of points $\left\{x_{i}\right\}$ of $S^{n}-B$, one from each component. Now from the duality for closed sets and (5.3), $C^{n-1}$ must be linked with the Cech-0-cycle on two of these points, say $x_{0}$ and $x_{1}$. But then $S^{n}-\left(x_{0} \cup x_{1}\right)$ is a neighborhood of $B$ in which $C^{n-1}$ is not $\cong 0$. Therefore, from Theorem $5, C^{n-1}$ is not $\sim 0$ in $B$.

CoROLlaRY 1. Let $B$ be an arbitrary subset of $S^{n}$. If the compact Cech cycles $C_{i}^{n-1}(i=1, \cdots, h)$ are independent relative to compact homologies in $B$, they are independent relative to general homologies in $B$.

Corollary 2. Let $A$ be an arbitrary subset of $S^{n}$, and let $S^{n}-A$ contain $h$ compact Cech-(n-1)-cycles independent there relative to compact homologies. Then $A$ contains $h$ compact $C$ ech-0-cycles independent in $A$ relative to general

(23) This is actually a corollary of a theorem of Eilenberg. Cf. [5]. 
homologies (or, what is the same thing, $A$ contains at least $h+1$ quasi-components) $\left({ }^{24}\right)$.

We close this section with some miscellaneous theorems. Our dimensions in the following are Menger-Urysohn dimensions.

(5.5) If a separable metric space $R$ is of dimension not greater than $k$, every covering of $R$ has a countable star-finite refinement of dimension not greater than $k$.

For a proof of this, see [8, Theorem VI]. The proof there is for finite coverings, but it easily extends to the countable star-finite case.

THEOREM 10. If an arbitrary subset $A$ of $S^{n}$ is of dimension not greater than $k$, every compact Cech-(n-k-2)-cycle in $S^{n}-A$ is $\cong 0$ there. In fact, for every compact subset $F_{0}$ of $S^{n}-A$, there is a compact $F, F_{0} \subset F \subset S^{n}-A$, such that all the Cech-(n-k-2)-cycles of $F_{0}$ bound in $F$.

Proof. From (5.5) above, every covering of $A$ has a refinement with no $(k+1)$-simplexes and therefore no $(k+1)$-cycles. Therefore $A$ is $(k+1)$-connected and the theorem follows from Theorem 7.

(5.6) If $A$ is homeomorphic to a subset of the Euclidean $k$-space $R^{k}, A$ is $k$-connected. In fact, every covering of $A$ has a refinement with no $k$-cycles.

Proof. We can assume $A \subset R^{k}$. Let $\mathfrak{u}$ be an arbitrary internal covering of $A$ and $\mathfrak{u}^{\prime}(G)$ a derived neighborhood covering. Choose a subdivision covering $\mathfrak{B}^{\prime}(G)=\left\{V_{\mathfrak{l}}^{\prime}\right\}$ which is $>\mathfrak{U}^{\prime}(G) \cdot \mathfrak{B}^{\prime}(G)$ has no $k$-cycles, since each of its cycles is the image of an actual polyhedral cycle in $G$, and the latter contains none. Then $\mathfrak{B}=\left\{V_{\boldsymbol{i}}^{\prime} \cap A\right\}$ is an internal covering of $A$ which is $>\mathfrak{U}$ and has no $k$-cycles.

THEOREM 11. If an arbitrary subset $A$ of $S^{n}$ is homeomorphic to a subset of the Euclidean $k$-space $R^{k}$, then for $j \leqq n-k-1$, every compact Cech-j-cycle of $S^{n}-A$ is $\cong 0$ outside of $A$. In fact, for every compact subset $F_{0}$ of $S^{n}-A$, there is a compact $F, F_{0} \subset F \subset S^{n}-A$, such that all the Cech-j-cycles of $F_{0}$ bound in $F$.

This follows from (5.6) above and Theorem 7.

We shall call $A \subset S^{n}$ Čech-k-accessible relative to compact homologies at a point $x$ of $A$, if every Cech- $k$-cycle in $S^{n}-A$ is $\cong 0$ in $\left(S^{n}-A\right) \cup x$.

(5.7) If $A$ is an arbitrary subset of $S^{n}$ and every proper subset of $A$ is $k$-connected, then $A$ is Cech-(n-k-1)-accessible relative to compact homologies at every point.

This follows from Theorem 7. From Theorem 10 we have:

(24) Cf. [5]. 
(5.8) An arbitrary subset of $S^{n}$ of dimension not greater than $k$ is Cech-jaccessible relative to compact homologies at every point, for $j \leqq n-k-2$.

And from Theorem 11:

(5.9) If an arbitrary subset $A$ of $S^{n}$ is homeomorphic to a subset of $R^{k}, A$ is Cech-j-accessible relative to compact homologies at every point, for $j \leqq n-k-1$.

6. Some homology properties of finite coverings. In Theorem 9, we showed that if $B$ is an arbitrary subset of $S^{n}$, then for the compact Cech$(n-1)$-cycles of $B$, bounding in the family of all coverings (that is, general bounding) is equivalent to compact bounding. We shall now show that we can restrict ourselves to finite coverings for this dimension. We first prove a preliminary theorem.

Theorem 12. Let $K$ be $a(k+1)$-dimensional polyhedral complex and $C^{k} a$ Cech cycle on $K$. If $C^{k} \sim 0$ in all finite coverings of $K$, it is $\sim 0$ in all coverings of $K$.

Proof. Assume $C^{k}$ is not $\sim 0$ in $K$. We shall produce a finite covering in which $C^{k}$ fails to bound. Let $C^{k}=\left\{Z^{k}(\mathfrak{u})\right\}$. If $\mathfrak{u}$ is any subdivision of $K$, then by $z^{k}(\mathfrak{u})$ we shall mean the image in $\mathfrak{u}$ of $Z^{k}(\mathfrak{U})$, where $\mathfrak{U}$ is the subdivision covering derived from $\mathfrak{u}$ and $Z^{k}(\mathfrak{U})$ is the coordinate of $C^{k}$ in $\mathfrak{U}$.

Lemma. Let $\mathfrak{v}$ be a subdivision of the $k$-sphere $S^{k}$ and $z_{s}^{k}(\mathfrak{b})$ the essential $k$-cycle of $\mathfrak{v}$. Then there is a subdivision $\mathfrak{u}$ of $K$ which can be mapped simplicially into $\mathfrak{v}$ so that the map of $z^{k}(\mathfrak{u})$ is $z_{\mathfrak{s}}^{k}(\mathfrak{b})$.

Proof. Hopf has proved this for a finite polyhedral complex [6], but his proof applies directly to an infinite one, and we repeat it here, adapted to our purpose. Since $C^{k}$ is not $\sim 0$ in $K$, there is a subdivision covering $\mathfrak{U}_{0}$ such that $Z^{k}\left(\mathfrak{U}_{0}\right)$ is not $\sim 0$ in $\mathfrak{U}_{0}$. Let $\mathfrak{u}_{0}$ be the subdivision from which $\mathfrak{U}_{0}$ is derived; then $z^{k}\left(\mathfrak{u}_{0}\right)$ is not $\sim 0$ in $\mathfrak{u}_{0}$.

Let $y_{i}^{k}\left(\mathfrak{u}_{0}\right)(i=1,2, \cdots)$ be a homology basis for the non-bounding $k$-chains of $\mathfrak{u}_{0}$. That is, no finite linear combination of the $y_{\mathfrak{i}}^{\boldsymbol{k}}\left(\mathfrak{u}_{0}\right)$ bounds and every $k$-chain $y^{k}\left(\mathfrak{u}_{0}\right)$ of $\mathfrak{u}_{0}$ is homologous to a unique linear combination of them: $y^{k}\left(\mathfrak{u}_{0}\right) \sim \sum c_{i} y_{\mathfrak{k}}^{k}\left(\mathfrak{u}_{0}\right)$ (only a finite number of the $c_{i}$ different from 0 ). We can choose $z^{k}\left(\mathfrak{u}_{0}\right)$ for our $y_{1}^{k}\left(\mathfrak{u}_{0}\right)$. We now define a homomorphism $H$ of the $k$-chains of $\mathfrak{u}_{0}$ in to the integers modulo 2 as follows:

$$
H\left(\sum c_{i} y_{i}^{k}\left(\mathfrak{u}_{0}\right)\right)=\left\{\begin{array}{lll}
1 & \text { if } & c_{1} \neq 0, \\
0 & \text { if } & c_{1}=0 .
\end{array}\right.
$$

$H$ has the following two properties:

(i) $H\left(y_{1}^{k}\left(\mathfrak{u}_{0}\right)\right)=1$,

(ii) If $y^{k}\left(\mathfrak{u}_{0}\right)$ is a bounding cycle, $H\left(y^{k}\left(\mathfrak{u}_{0}\right)\right)=0$.

Let $K^{k}$ be the subcomplex of $K$ obtained by dropping out all the $(k+1)$ - 
simplexes. We define a mapping $f_{0}$ of $K^{k}$ into $S^{k}$ as follows: Choose one of the vertices $p$ of the subdivision $\mathfrak{b}$ of $S^{k}$ and let the boundary of each $k$-simplex $e^{k}$ of $K$ be mapped into $p$. If $H\left(e^{k}\right)=0$, map the interior of $e^{k}$ also into $p$; if $H\left(e^{k}\right)=1$, map the interior homeomorphically into $S^{k}-p$. In this way, the degree of mapping of $e^{k}$ is exactly $H\left(e^{k}\right)$. Now every $k$-chain $y^{k}\left(\mathfrak{u}_{0}\right)$ is a finite linear combination of the $e^{k}: y^{k}=\sum a_{j} e_{j}^{k}$. Hence

$$
H\left(y^{k}\left(\mathfrak{u}_{0}\right)\right)=H\left(\sum a_{j} e_{j}^{k}\right)=\sum a_{j} H\left(e_{j}^{k}\right) .
$$

Since the degrees of mapping add algebraically in this same fashion, it follows that the degree of mapping of $y^{k}\left(\mathfrak{u}_{0}\right)$ is exactly $H\left(y^{k}\left(\mathfrak{u}_{0}\right)\right)$. In particular, from (i):

(iii) $y_{1}^{k}\left(\mathfrak{u}_{0}\right)$ maps into $S^{k}$ with degree 1 .

We extend $f_{0}$ to all of $K$ as follows. Let $e^{k+1}$ be any $(k+1)$-simplex of $\mathfrak{u}_{0}$ and $\partial e^{k+1}$ its boundary. From (ii), $H\left(\partial e^{k+1}\right)=0$. Therefore $\partial e^{k+1}$ maps into $S^{k}$ with degree zero, and hence $f_{0}$ can be extended to $e^{k+1}$.

We now vary $f_{0}$ slightly to obtain our required mapping $f$. By the fundamental deformation theorem, there is a refinement $\mathfrak{u}$ of $\mathfrak{u}_{0}$ and a homotopic deformation of $f_{0}$ over $S^{k}$ such that no point leaves the closed simplex of $\mathfrak{v}$ containing it and such that when we are through, we have a simplicial mapping of $\mathfrak{u}$ into $\mathfrak{b}$. This is our final mapping $f$. Under it, the part of $K$ which was mapped into $p$ remains there, and the degree of mapping of each chain remains unchanged. In particular, if $y^{k}(\mathfrak{u})$ is the refinement in $\mathfrak{u}$ of $y_{\mathfrak{k}}^{\mathfrak{k}}\left(\mathfrak{u}_{0}\right)$, then, from (iii), $y^{k}(\mathfrak{u})$ maps into $S^{k}$ with degree 1 . But, since $y_{\mathfrak{1}}^{k}\left(\mathfrak{u}_{0}\right)=z^{k}\left(\mathfrak{u}_{0}\right)$, we must have $y^{k}(\mathfrak{u}) \sim z^{k}(\mathfrak{u})$ in $\mathfrak{u}$. Therefore $z^{k}(\mathfrak{u})$ maps into $S^{k}$ with degree 1 . It follows that

$$
f\left(z^{k}(\mathfrak{u})\right)=z_{\mathfrak{s}}^{k}(\mathfrak{y}),
$$

which completes the proof of the lemma.

Returning to the theorem, let $\mathfrak{B}=\left\{V_{i}\right\}$ be the subdivision covering of $S^{k}$ derived from $\mathfrak{v}$, and denote the image of $z_{s}^{\mathfrak{k}}(\mathfrak{b})$ by $Z_{s}^{\mathfrak{k}}(\mathfrak{B})$. Since $S^{k}$ is a finite complex, $\mathfrak{B}$ is finite. Then $\mathfrak{B}^{-1}=\left\{V_{i}^{-1}\right\}$, where $V_{i}^{-1}$ is the complete inverse of $V_{\mathfrak{i}}$ under $f$, is a finite covering of $K$. Further, $\mathfrak{u}$, the subdivision covering of $K$ derived from $\mathfrak{u}$, is a refinement of $\mathfrak{B}^{-1}$. For, since $f$ maps $\mathfrak{u}$ simplicially into $\mathfrak{v}$, the star of each vertex of $\mathfrak{u}$ maps into the star of some vertex of $\mathfrak{v}$; that is, each element of $\mathfrak{U}$ maps into some element of $\mathfrak{B}$. It follows that each element of $\mathfrak{u}$ lies in some element of $\mathfrak{B}^{-1}$. If we denote the inverse of $Z_{\mathfrak{s}}^{\mathfrak{k}}(\mathfrak{B})$ by $Z_{s}^{\mathfrak{k}}\left(\mathfrak{B}^{-1}\right)$, it is easily shown from (iv) that $Z^{k}(\mathfrak{U})$ projects into $Z_{s}^{k}\left(\mathfrak{B}^{-1}\right)$. But $Z_{s}^{\mathfrak{k}}\left(\mathfrak{B}^{-1}\right)$ is not $\sim 0$ in $\mathfrak{B}^{-1}$. Hence $C^{k}$ fails to bound in the finite covering $\mathfrak{B}^{-1}$, and the theorem is proved.

THEOREM 13. Let $B$ be an arbitrary subset of $S^{n}$ and $C^{n-1} a C_{e c h-(n-1)-}$ cycle of $B$. Then if $C^{n-1} \sim 0$ in all finite converings of $B, C^{n-1} \sim 0$ in all coverings of $B$. 
Proof. We need only consider neighborhood coverings of $B$. Let $G$ be a neighborhood of $B$. Since $C^{n-1} \sim 0$ in all finite coverings of $G$, we have from Theorem 12 that $C^{n-1} \sim 0$ in all coverings of $G$. But $G$ is any neighborhood of $B$. Therefore $C^{n-1} \sim 0$ in all neighborhood coverings of $B$.

THEOREM 14. Let $B$ be an arbitrary subset of $S^{n}$ and $C^{n-1}$ a compact Cech$(n-1)$-cycle of $B$. Then if $C^{n-1} \sim 0$ in all finite coverings of $B, C^{n-1} \cong 0$ in $B$.

This follows from Theorem 13 and Theorem 9.

In Theorem 2 we gave a necessary and sufficient condition that a compact Cech cycle $C^{k}$ in a separable metric space $R$ bound in all coverings of $R$, namely, if $V^{k}$ is a Vietoris counterpart of $C^{k}, V^{k}$ null-bounds under all homeomorphic remetrizations of $R$. We shall now give an analogous equivalent condition for $C^{k}$ to bound in all finite coverings of $R$.

TheOREM 15. Let $R$ be a separable metric space, $C^{k}=\left\{Z^{k}(\mathfrak{U})\right\}$ a compact Cech cycle in $R$, and $V^{k}=\left\{Z_{m}^{k}\right\}$ a counterpart Vietoris cycle. Then a necessary and sufficient condition that $C^{k} \sim 0$ in all finite coverings of $R$ is that $V^{k} \sim 00$ under all totally bounded metrics imposed on $R$ (which do not change the topology).

Proof. Assume $C^{k} \sim 0$ in all finite coverings and suppose $R$ has a totally bounded metric. To show that $V^{k} \sim \sim_{0} 0$ in $R$, we need to show that for arbitrary $\epsilon>0, V^{k} \sim, 0$ in $R$. Given $\epsilon>0$, from the total boundedness, there is a finite $\epsilon / 3$-covering of $R$. We then proceed exactly as we did in proving the necessity in Theorem 2.

Now assume there is some finite covering $\mathfrak{U}=\left\{U_{i}\right\}$ such that $Z^{k}(\mathfrak{u})$ is not $\sim 0$ in $\mathfrak{U}$. From (1.1) we can assume $\mathfrak{U}$ is a closure-isomorphic covering. Since we can take $R$ imbedded in the Hilbert cube, we can assume that its given metric is totally bounded. We shall obtain a new metric, also totally bounded, in which $V^{k}$ is not $\sim_{0} 0$. We shall use the following theorem of Hurewicz [7].

(6.1) Let $R$ be a metric space and let $\left\{E_{m}\right\}$ be a finite collection of closed sets, $E_{m}=F_{1}^{(m)}, \cdots, F_{h}^{(m)}(m=1, \cdots, M)$, such that $\bigcap_{i=1}^{h(m)} F_{i}^{(m)}=0$ for each $m$. Then for every $\epsilon>0$, we can remetrize $R$ so that:

(a) $0 \leqq \rho_{1}(x, y)-\rho(x, y) \leqq \epsilon$, where $\rho$ is the original and $\rho_{1}$ the new metric;

(b) For each $E_{m}$, there is a $\delta_{m}>0$ such that every set of points $x_{i} \in F_{i}^{(m)}$ $(i=1, \cdots, h(m))$ is of diameter greater than $\delta_{m}$;

(c) If $R$ is totally bounded under $\rho$, it is totally bounded under $\rho_{1}$.

Let $\left\{E_{m}\right\}(m=1, \cdots, M)$ be the collection of all $(k+2)$-tuples of $\left\{U_{i}\right\}$, such that if $E_{m}=\left\{U_{i(j)}\right\}(j=0, \cdots, k+1), \bigcap_{j=0}^{k+1} \bar{U}_{i(j)}=0$. Since $U$ is finite, there are only a finite number of the $E_{r}$, so we can apply (6.1) and obtain a new metric $\rho_{1}$. Take $\delta<\min \left(\delta_{1}, \cdots, \delta_{M}\right)$. Then, as in Theorem 2 (see (ix) there), we have the following property: 
If the points $x_{0}, \cdots, x_{k+1}$ form a set of diameter less than $\delta$ and $x_{j} \in U_{i(j)}$ $(j=0, \cdots, k+1)$, then $\bigcap_{j=0}^{k+1} U_{i(j)} \neq 0$.

Then proceeding as we did in Theorem 2 , we can show that $V^{k}$ is not $\sim_{0} 0$.

\section{REFERENCES}

1. P. Alexandroff, Untersuchungen ïber Gestalt und Lage abgeschlossener Mengen beliebiger Dimension, Ann. of Math. vol. 30 (1928) pp. 101-187.

2. P. Alexandroff and H. Hopf, Topologie, vol. 1, Berlin, 1935.

3. E. Cech, Theorie generale de l'homologie dans un espace quelconque, Fund. Math. vol. 19 (1932) pp. 149-183.

4. C. H. Dowker, Mapping theorems in non-compact spaces, Princeton Thesis, 1938.

5. S. Eilenberg, An invariance theorem for subsets of $S^{n}$, Bull. Amer. Math. Soc. vol. 47 (1941) pp. 73-75.

6. H. Hopf, Über Abbildungen der dreidimensionalen Sphäre auf der Kugelflache, Math. Ann. vol. 104 (1931) pp. 637-666.

7. W. Hurewicz, Über Einbettung separabler Raume in gleichdimensionale kompakte Raume, Monatshefte für Mathematik und Physik vol. 37 (1930) pp. 199-209.

8. W. Hurewicz and H. Wallman, Dimension theory, Princeton University Press, 1941.

UNIVERSITY OF MICHIGAN, ANn ARbor, Mich. 